\title{
The variable X-ray spectrum of Markarian 766
}

\section{Time-resolved spectroscopy $\star, \star \star$}

\author{
T. J. Turner ${ }^{1,2}$, L. Miller ${ }^{3}$, J. N. Reeves ${ }^{4}$, and S. B. Kraemer ${ }^{2,5}$ \\ 1 Dept. of Physics, University of Maryland Baltimore County, 1000 Hilltop Circle, Baltimore, MD 21250, USA \\ e-mail: turner@milkyway.gsfc.nasa.gov \\ 2 Astrophysics Science Division, NASA/GSFC, Greenbelt, MD 20771, USA \\ 3 Dept. of Physics, University of Oxford, Denys Wilkinson Building, Keble Road, Oxford OX1 3RH, UK \\ 4 Astrophysics Group, School of Physical and Geographical Sciences, Keele University, Keele, Staffordshire ST5 5BG, UK \\ 5 Department of Physics, Catholic University of America, Washington DC 20064, USA
}

Received 25 May 2007 / Accepted 10 August 2007

ABSTRACT

\begin{abstract}
Context. The variable X-ray spectra of AGN systematically show steep power-law high states and hard-spectrum low states. The hard, low state has previously been found to be a component with only weak variability. The origin of this component and the relative importance of effects such as absorption and relativistic blurring are currently not clear.

Aims. In a follow-up of previous principal components analysis we aim to determine the relative importance of scattering and absorption effects on the time-varying X-ray spectrum of the narrow-line Seyfert 1 galaxy Mrk 766.

Methods. Time-resolved spectroscopy, slicing XMM and Suzaku data down to 25 ks elements is used to investigate whether absorption or scattering components dominate the spectral variations in Mrk 766.

Results. Time-resolved spectroscopy confirms that spectral variability in Mrk 766 can be explained by either of two interpretations of principal components analysis. Detailed investigation confirm rapid changes in the relative strengths of scattered and direct emission or rapid changes in absorber covering fraction provide good explanations of most of the spectral variability. However, a strong correlation between the $6.97 \mathrm{keV}$ absorption line and primary continuum together with rapid opacity changes show that variations in a complex and multi-layered absorber, most likely a disk wind, are the dominant source of spectral variability in Mrk 766.
\end{abstract}

Key words. galaxies: Seyfert - X-rays: galaxies

\section{Introduction}

Determination of the origin of X-ray spectra of active galactic nuclei has been mired in ambiguity with different classes of candidate models predicting similar time-averaged spectra. A continuum in the form of a power-law is at the core of all popular models, a "reflection spectrum" produced by scattering from the surface of Compton-thick material is also likely to be present. The putative accretion disk (Guilbert \& Rees 1988; Lightman \& White 1988; George \& Fabian 1991) must exist near the continuum region and such reflection would produce a hard spectral component with strong Fe $\mathrm{K}$ emission, observable below $10 \mathrm{keV}$ (George \& Fabian 1991; Zdziarski et al. 1995; Perola et al. 2002; Tanaka et al. 1995; Nandra et al. 1997). Layers of Compton-thin gas are thought to shroud the nuclear system, with strong and (relatively) unambiguous evidence for the presence of zones covering a large range of ionization-states and column densities (Crenshaw et al. 2003). If "competing" models have the same constituents then what makes them different? The principal distinction is the degree to which reflection processes dominate versus absorption. Also of general interest is whether

\footnotetext{
* Based on observations obtained with XMM-Newton, an ESA science mission with instruments and contributions directly funded by ESA Member States and NASA, also on observations obtained with Suzaku, a collaboration between ISAS/JAXA and NASA/GSFC, MIT.

$\star \star$ Appendix A is only available in electronic form at http://www . aanda.org
}

we can detect the signature of relativistic blurring in the reprocessed components. In practice, models dominated by blurred reflection are indistinguishable from those composed of layers of complex absorption. It has proved difficult to progress with current data, where one typically has a short baseline for the observation and is limited to model-fitting time-averaged X-ray spectra with only modest energy resolution (Reeves et al. 2004; Turner et al. 2005).

A possible way forward is to use the observed spectral changes over time to break the ambiguity. Rather than simply fitting models to time-averaged data we can attempt to disentangle components that arise on different physical scales and in different zones by their differing patterns of spectral variability. Marked spectral variability is indeed observed in local AGN down to timescales of thousands of seconds, and the long observations of these sources that have been performed recently yield good sampling of spectral variability and the possibility of isolating the dominant effects.

Very long observations of AGN that tend to only be performed once a mission is well-established, have turned out to be invaluable because exposure times $>$ a day have pushed us into a regime where new results have been observable. For example, a long XMM observation of Mrk 766 has shown that the primary variable component of the spectrum has the form of an absorbed power-law with accompanying ionized reflection that varies with the continuum. The latter result first became evident with the discovery of the correlation between ionised line 
emission and continuum variations down to $10 \mathrm{ks}$ (Miller et al. 2006). These results also concur with the analysis of the 2001 data by Turner et al. (2006) where evidence for a periodic Doppler shift of ionised emission from a radius $r \sim 100 r_{\mathrm{g}}$ was found.

Available long datasets also inspired the first application of principal components analysis (PCA) to data from a single X-ray observation (Vaughan \& Fabian 2004). By correlating flux at different spectral energies one can decompose data into variable constituent components, this method works well to break data into mathematically "additive" components (emission components, direct or reprocessed) but has a limitation because it cannot separate out the mathematically "multiplicative" changes that would be caused, for example, by varying absorption. The conclusion from the Vaughan \& Fabian (2004) PCA study of MCG-6-30-15 was that the spectral variability in that case was driven primarily by a power-law of varying normalization combined with a slowly-varying or constant hard component of reflection.

In the first paper in this series (Miller et al. 2007) we investigated spectral variability in $X M M$ observations of Mrk 766, a narrow-line Seyfert 1 galaxy at redshift $z=0.0129$ (Osterbrock \& Pogge 1985). We applied a PCA method based on singular value decomposition that preserved the full instrumental spectral resolution. It was found that the source can be modeled adequately using just two additive spectral components: a variable component comprising a power-law and ionised, modestlybroadened $\mathrm{Fe}$ emission line together with relatively non-variable component(s) that shows a hard spectral form.

The possible origins for the hard component have been discussed at length in Paper I. A model where there is some scattered and self-absorbed fraction of the primary continuum and possibly a contribution from a distant neutral reflector can explain the offset component shape and the spectral variability (hereafter the "scattering" model). Alternatively, the hard flux could be a heavily-absorbed fraction of the continuum, and we denote this the "partial-covering absorption" model ("p-cov absorption"). In the latter model the PCA breakdown into additive components indicated that covering fraction changes produce the observed spectral variability, as the additive components do not change much in shape but rather in relative dominance as the source flux varies. Importantly, both these interpretations of the PCA work without recourse to relativistic blurring of any spectral component. Miller et al. (2007) discussed fits that include blurred reflection and concluded that significant blurring was not required to explain Mrk 766, although the source is consistent with the presence of such effects.

Here, we present here a detailed analysis of time-resolved spectra of Mrk 766, fitting spectral models to individual time intervals. $X M M$ has accumulated $\sim 700 \mathrm{ks}$ of data from this highlyvariable Seyfert galaxy from 2000-2005. The XMM data discussed here have already been used to detect variability of the peak energy and flux of a somewhat broad, ionised Fe emission line in the 6-7 keV range (Miller et al. 2006). Here we show, for the first time, the PCA model fit to the data themselves, comparing the two physical interpretations of the PCA results presented by Miller et al. (2007), i.e. the scattering and p-cov absorption models, to understand whether scattering/reflection or absorption effects are dominant. Of particular interest here is a followup of the time-dependent behavior of two absorption features found in the Fe K-band using PCA, a feature at $6.97 \mathrm{keV}$ likely originates in $\mathrm{H}$-like $\mathrm{Fe}$ while a feature at $7.3 \mathrm{keV}$ is of uncertain origin.
In this paper, we concentrate on the origin of spectral variability in Mrk 766. In this sense, consideration of what we dub the "scattering model" may be taken as including consideration of a relativistically blurred reflection model, since the scattered component may also be fitted by such a model (Miller et al. 2007), although we note in Sect. 6 how absorption line variations may be used to distinguish relativistically-blurred from non-blurred models. Whatever the model fit to the "hard" component, its temporal behavior is a key piece of information that may help us disentangle the possible explanations for its origin. In addition to this new treatment of the $X M M$ data we present new Suzaku data allowing us to extend the analysis to $\sim 50 \mathrm{keV}$, and increase the temporal baseline for the study.

\section{The data}

\subsection{XMM-Newton data}

In this paper we utilise all XMM-Newton (hereafter XMM) European Photon Imaging Camera (EPIC) pn CCD (Strüder et al. 2001) data available for Mrk 766, covering 2000 May 20 (observation ID 0096020101), 2001 May 20-21 (0109141301) and 2005 May 23 (0304030[1-7]01). The data reduction is described by Miller et al. (2006) and as in that work, the combination of some non-optimal modes for the Metal Oxide SemiConductor CCDs, photon pileup and inferior signal-to-noise led us to use only the pn CCD data for analysis.

To perform time-resolved spectroscopy we found that timeselections of $25 \mathrm{ks}$ duration gave adequate signal-to-noise in all spectra (slightly lower effective exposure times in some cases are due to data gaps). This sampling gave a good match to the variability timescale evident in the data. Where datasets did not divide exactly into $25 \mathrm{ks}$ bins, the final bin of an orbital segment was allowed to be slightly shorter or longer than this. This selection criterion yielded 28 spectral slices in total; 2000 May yielded two slices, 2001 yielded five slices and 2005 yielded 21 slices. With time-sliced spectra, despite our attempts to optimize the selections, the signal-to-noise at high energies, $E>7.5 \mathrm{keV}$, was still found to be low. To optimize the spectral fitting we binned all spectra consistently with energy bins equal in width to the Half Width Half Maximum (HWHM) spectral resolution of the EPIC pn instrument. The HWHM varies with photon energy, so the bin widths chosen also vary continuously with energy, from $\sim 35 \mathrm{eV}$ at $1 \mathrm{keV}$ to $\sim 80 \mathrm{eV}$ at $10 \mathrm{keV}$.

The energy range chosen for the analysis initially is $1.0-9.8 \mathrm{keV}$, this focuses the study on gas closest to the nucleus and avoids modeling in detail emission/absorption lines below $1 \mathrm{keV}$, some of which likely arise from gas relatively distant from the central engine (e.g. Ogle et al. 2003). The truncation at $9.8 \mathrm{keV}$ is where signal-to-noise reaches a very low level.

\subsection{Suzaku data}

Mkn 766 was observed by Suzaku from 2006 November 16 00:35 to 2006 November 18 08:44 in XIS nominal mode. We used events files from version 1.3.2.6 of the Suzaku pipeline. The XIS data was reduced using v.6.1.2 of the "ftools" package. It was screened with XSELECT to exclude data within the South Atlantic Anomaly (SAA) and data within $436 \mathrm{~s}$ of the SAA. Additionally we excluded data with an Earth elevation angle less than $5^{\circ}$ and Earth day-time elevation angles less than $20^{\circ}$. Finally we selected good events with grades $0,2,3,4$, and 6 and removed hot and flickering pixels using the SISCLEAN script. This screening left us with effective exposures of $\sim 87 \mathrm{ks}$ for each of the XIS 0,1 , and 3 chips. Note that the spaced-row charge injection (SCI) was not active during this observation. 
The XIS products were extracted from circular regions of 2.9' while background spectra were extracted from a region of the same size offset from the source (and avoiding the chip corners with the calibration sources). The response and ancillary response files were then created using the tasks XISRMFGEN and XISSIMARFGEN, respectively. The source contributed $98.6 \%$, $95.3 \%$, and $98.4 \%$ of the total counts in from the XIS 0,1 , and 3 products, respectively. The orbit of Suzaku leads to the exposure noted above being spread out over a $200 \mathrm{ks}$ baseline. To obtain time slices with similar exposure to the $X M M$ spectra we split the data into four equal time periods covering a baseline of $50 \mathrm{ks}$ each, for spectral analysis.

The PIN background events file was provided by the HXD instrument team, used in conjunction with the source events file to create a good time interval applicable to both the source and background. The background events file was generated using ten times the actual background count rate, so we increased the effective exposure time of the background spectra by a factor of 10. We found the deadtime correction factor using the HXDDTCOR task with the extracted source spectra and the unfiltered source events files. After deadtime correction, we were left with an effective exposure of $\sim 87 \mathrm{ks}$. To take into account the cosmic X-ray background (Boldt \& Leiter 1987; Gruber et al. 1999) XSPEC v 11.3.2 Arnaud (1996) was used to generate a spectrum from a CXB model, normalized to the Suzaku field of view, and combined with the PIN simulated background file using MATHPHA to create an total background file. The source comprised $6.7 \%$ of the total counts.

\section{The models}

In this paper, as we have many spectral components in the fits, we refer to the ionised reflection that is viewed directly (and appears from the variability of the Fe K emission line to correlate with the directly-viewed powerlaw) as the "ionised reflector". We denote the offset component to be the "hard" or "scattered" component. It is not clear whether the scattering gas is Comptonthick in that case and the use of the term "scattered" allows an easy distinction between this steady component and the ionised reflector that is correlated with the continuum flux. Miller et al. (2007) also considered whether models where the spectral variability was dominated by absorption changes might explain the data, concluding that the hard component could be described as an absorbed fraction of the primary continuum and that changes in covering fraction could then explain the source behavior. As described in the introduction we term this the "p-cov absorption" model. Both "scattering" and "p-cov absorption" models are consistent with the PCA provided the spectral variability is dominated by covering fraction changes in the p-cov absorption model.

The general approach taken was to utilize the fits to the direct and offset components from the application of PCA to the $X M M$ data (Miller et al. 2007), then extend the analysis to include the broader bandpass Suzaku data, as a test of the model on data not used to create it.

Miller et al. (2007) fitted the spectral elements derived from PCA, to investigate the source in terms of additive spectral components. Here we fit the data directly using time-resolved spectroscopy and this allows us to test how well the two PCA interpretations fit each time period for Mrk 766, and to see how the different model parameters vary with time. This analysis also aims to expand upon the PCA by finding an explanation for the residual spectral variability that was not well modeled there. As noted above then, we apply two interpretations of the PCA

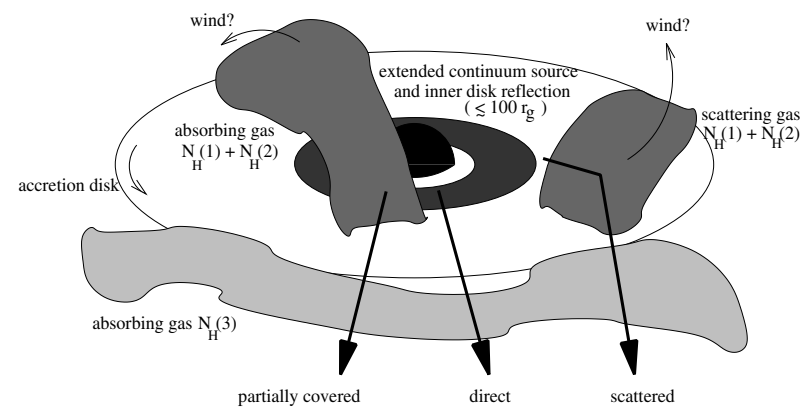

Fig. 1. A schematic representation of the inner regions of the models described in Sect. 3. The distant reflector is omitted from the scattering model, for clarity.

decomposition, first we fit the "scattering model" and then the "p-cov absorption model" that uses varying covering fraction to explain the source. The derivation of these models from the PCA is discussed in detail by Miller et al. (2007), and so the two models to be utilized are simply summarized below. Here we use the $\xi$ form of ionization parameter where $\xi=L / n r^{2}, L$ is the $1-1000$ Rydberg luminosity, $n$ the gas density and $r$ the absorber-source distance. We quote $\xi$ in units of erg $\mathrm{cm} \mathrm{s}^{-1}$ throughout.

\subsection{The scattering model}

The scattering model (Fig. 1) is that found from fitting the PCA hard component using the scattered primary continuum (whose slope was determined from fitting the PCA variable component). The full spectral model then consists of:

1. a power-law of constant slope $\Gamma=2.38$ and variable normalization;

2. an ionised reflector that varies with normalization tied to that of the power-law;

3. a constant scattered component. This is represented by summing components 1 and 2 and then allowing a scalar to represent the scattered fraction;

4. a complex layer of ionised absorption in front of the scattered component, represented by the cmbination of two layers: $N_{\mathrm{H}}(1)=4.74 \times 10^{22} \mathrm{~cm}^{-2}, \log \xi(1)=-0.17, N_{\mathrm{H}}(2)=$ $3.97 \times 10^{23} \mathrm{~cm}^{-2}, \log \xi(1)=3.05$. This physically might arise if the reflecting/scattering surface has its own atmosphere, for example;

5. a neutral reflector with no blurring using the XSPEC pexrav model with a Gaussian emission line representing $\mathrm{Fe} \mathrm{K} \alpha$ at the strength expected for a slab of material inclined at $30^{\circ}$ (George \& Fabian 1991);

6. a low-column, low-ionization absorber fully covering all components: $N_{\mathrm{H}}(3)=2.77 \times 10^{21} \mathrm{~cm}^{-2}, \log \xi(3)=1.80$ covered all components during 2000-2001 while $N_{\mathrm{H}}(3)=$ $2.41 \times 10^{21} \mathrm{~cm}^{-2}, \log \xi(3)=1.26$ covered all components during 2005 and during the 2006 Suzaku observation;

7. two Gaussian absorption lines, allowed to float in normalization, with energies fixed at $6.97 \mathrm{keV}$ and $7.3 \mathrm{keV}$ (in the rest-frame of the host galaxy).

Construction of this model assumed the highest state observed to date (in 2001) to represent the sum of directly viewed and reflected components; data from the lowest flux state observed in $X M M$ data dominated the determination of the form of the hard component, although a small contribution from the direct component was allowed in that determination: the initial parameters values were chosen from the model fits to the PCA components. 


\subsection{The p-cov absorption model}

The p-cov absorption model (Fig. 1) is determined using several layers of ionized gas to explain the overall shape of the hard component, this complex absorption profile for the absorbed fraction is consistent with many physical models. The model is composed of:

1. a power-law of constant slope $\Gamma=2.18$ and variable normalization;

2. an ionized reflector that varies with normalization tied to that of the power-law;

3. a large and complex layer of absorption composed of $N_{\mathrm{H}}(1)=4.03 \times 10^{22} \mathrm{~cm}^{-2}, \log \xi(1)=1.19$ overlaying a second layer $N_{\mathrm{H}}(2)=4.57 \times 10^{23} \mathrm{~cm}^{-2}, \log \xi(1)=2.3 \mathrm{com}-$ prised the complex absorber whose variable covering fraction accounts for much of the observed spectral variability. The column densities and ionisation parameters of these two layers were initially assumed to be constant throughout the time-resolved fitting for both models but later we found it necessary to allow $N_{\mathrm{H}}(1)$ to vary (Table 1 );

4. a narrow, neutral component of $\mathrm{Fe} \mathrm{K} \alpha$;

5. a low-column, low-ionisation absorber covers all components: $N_{\mathrm{H}}(3)=5.38 \times 10^{21} \mathrm{~cm}^{-2}, \log \xi(3)=2.27$ for 2000-2001 $N_{\mathrm{H}}(3)=3.91 \times 10^{21} \mathrm{~cm}^{-2}, \log \xi(3)=1.93$ for 2005-2006;

6. two Gaussian absorption lines, allowed to float in normalization, with energies fixed at $6.97 \mathrm{keV}$ and $7.3 \mathrm{keV}$ (in the rest-frame of the host galaxy).

Construction of this p-cov absorption model assumed the highest state observed to date to be uncovered with respect to this complex dense absorbing layer, while a low column, moderately ionized gas layer of component 5 covers the direct component (and all other components) at all times. The power-law index is flatter in this model than in the scattering model because in the latter the hard scattered component exists in all flux states, but it is absent in the p-cov absorption model. The high-state data thus determined the form of the underlying continuum before the variable covering by absorption is applied. The fits then allow any additive combinations of the uncovered and covered fractions to fit each spectral slice. The fact that almost all shape parameters are fixed means the model has very few degrees of freedom and the method of construction is designed to isolate the key variables most effectively. The uncovered and covered fractions are naturally anti-correlated with this construction, although they have not been strictly required to always sum to the same total intrinsic flux (i.e. some intrinsic variation in continuum normalization is allowed but not required).

\subsection{Fitting the models}

Thus, following the results from the PCA, the slope of the power-law in each model was frozen at the best fit value found in each scenario. The ratio of power-law to ionized reflector was also frozen for each model and the normalization of the summed pair 1 plus 2 allowed to vary; this reproduces the known line/continuum correlation (Miller et al. 2006). It was found that the low column, low-ionization layer of absorption needed to have a different column density for the 2000-2001 and the 2005 epochs, and the column density was fixed at the best-fit value for each of those two time periods, in each model run.

The 2005 value derived from $X M M$ was later found applicable to the $S u z a k u$ data and utilized in those fits.
In both cases, the ionized reflector was modeled using the "reflion" model of Ross \& Fabian (2005), assuming solar abundances and that $\xi$ was fixed at $1600 \mathrm{erg} \mathrm{cm} \mathrm{s}^{-1}$, as determined from fitting the mean spectrum. Some blurring was allowed in the reflection spectrum via a Laor et al. (1991) convolution model, provided in XSPEC by the KDBLUR function, this was a very modest blurring constrained to arise within $100-300 r_{\mathrm{g}}$ from a disk inclined at $30^{\circ}$ to the line-of-sight, and necessary to model the $\sigma \sim 0.27 \mathrm{keV}$ width of the ionized diskline.

Layers of absorption were modeled using tables generated from XSTAR version $21 \mathrm{ln}$ (Kallman et al. 2004) with solar abundances, and turbulent velocity $200 \mathrm{~km} \mathrm{~s}^{-1}$. The Gaussian model absorption lines at 6.97 and $7.3 \mathrm{keV}$ were included in addition to the complex absorption components as these were not modeled by those absorption layers. The $6.97 \mathrm{keV}$ line most likely arises from highly ionized gas and thus would have little or no signature at lower energies (unless the column density is enormous). The $7.3 \mathrm{keV}$ line is of uncertain identification and thus it is most safely modeled in an isolated manner.

For the scattering model, the initially-free components were the normalizations of the directly-viewed and scattered fractions of the summed powerlaw plus ionized reflector (i.e. 1 plus 2). Additional free components were: the normalization of an additional neutral component of reflection, modeled using the XSPEC pexrav model with a linked $\mathrm{Fe} \mathrm{K} \alpha$ line of strength expected from a neutral reflector subtending $2 \pi$ steradians to the illuminating source and viewed at $30^{\circ}$ (George \& Fabian 1991) and the normalizations of narrow Gaussian components to model absorption features at 6.97 and $7.3 \mathrm{keV}$ (in the rest-frame of the host galaxy).

For the p-cov absorption model the initial free components were the normalizations of the unabsorbed and absorbed fractions of the spectrum (by design, these are anti-correlated in this model); and the Gaussian line normalizations, as before. In this model the neutral $\mathrm{Fe}$ line at $6.4 \mathrm{keV}$ is not linked to a component of neutral reflection, but allowed to vary in isolation (this model assumes that line arises from transmission through the absorber and not from a neutral reflector).

The absorption lines, while highly significant in the mean $X M M$ spectrum and in the PCA offset component (Miller et al. 2007) are highly sensitive to the form of the spectral model, as they fall in a region of some complexity. For this reason, the line fluxes derived from each model differ slightly, and in the paper we present both sets of values for comparison. A close-up of the $\mathrm{Fe} \mathrm{K}$ region is shown in Fig. 2 for slice 9 of the $X M M$ data.

\section{Results}

\subsection{XMM results}

First fitting all 28 time slices of the $X M M$ data over $1-9.8 \mathrm{keV}$ in the observed frame, the scattering model gave a total goodnessof-fit $\chi^{2}=4796$ for 3752 degrees of freedom (d.o.f.) while the $\mathrm{p}$ cov absorption model gave 3785 for 3752 d.o.f.. The fits confirm that despite having relatively few varying components, both our two physical representations of the PCA components do indeed describe the spectral variability to first order, and the models describe many spectral slices completely satisfactorily (Fig. 3). Successful first-order application of the two physical models to the data leaves us in a position to now determine the origin of the additional complexity that could not be accounted for from the PCA. This is a crucial step because we need to achieve a statistically superior set of fits before a meaningful comparison of models can be made. To this end, we examined the 


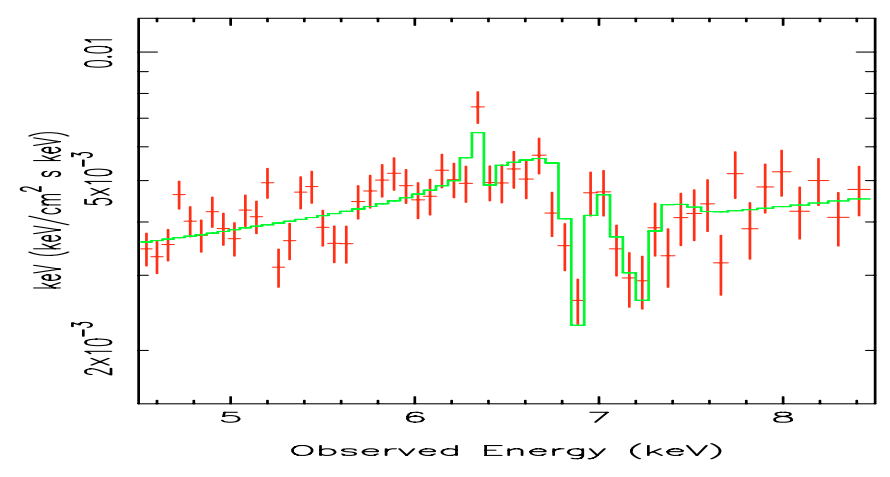

Fig. 2. A p-cov absorption fit to a slice of the low-state XMM data (slice 9) illustrating the presence of the two absorption features, at $6.97 \mathrm{keV}$ and $7.3 \mathrm{keV}$ in the rest-frame of the host galaxy.
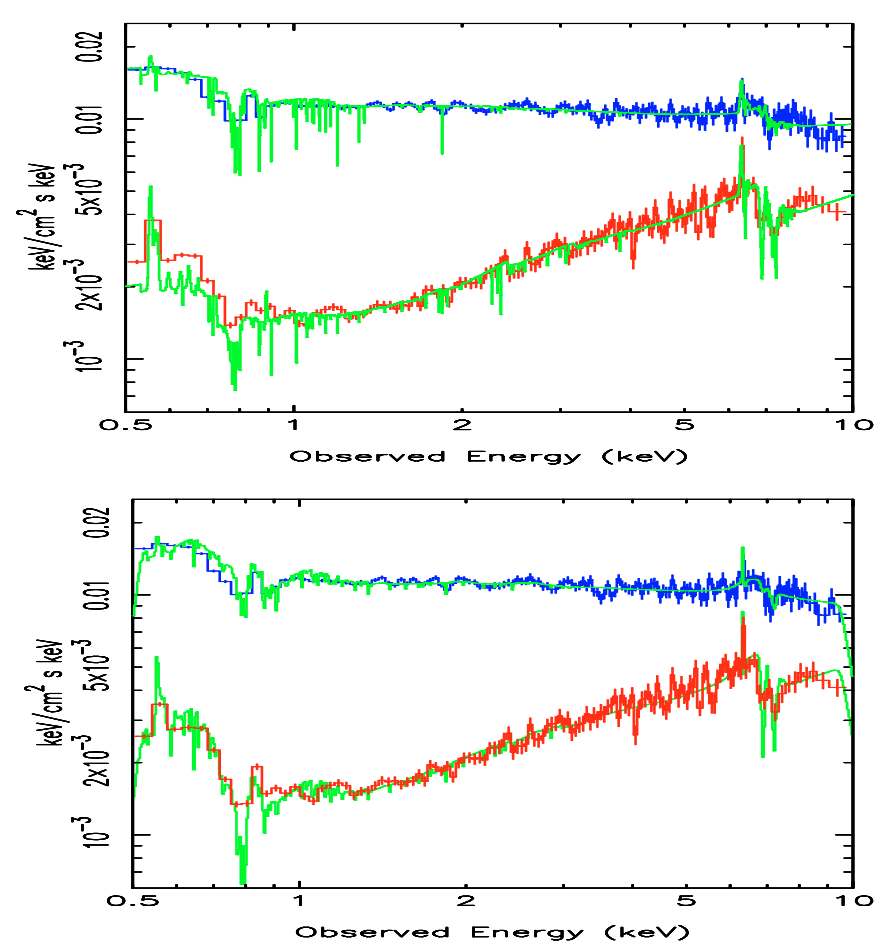

Fig. 3. Scattering model (top) and p-cov absorption model (bottom) fits are shown (solid green lines). The model lines represent fits to two of the individual time slices of XMM-Newton data (slice 6 is blue, slice 9 is red).

residuals to the worst fits, finding that allowing a change in column density of one of the zones of absorption can explain the remaining residuals. Allowing this extra degree of freedom significantly improves fits to both models. This makes sense, since the PCA could not handle multiplicative changes to the source spectrum (note that covering fraction changes alone would show up as additive changes in PCA, since the profile doesn't change but rather the amount of covered and uncovered source spectrum) and one might thus expect that a multiplicative variation would be the origin of spectral variability not handled by PCA. The column density changes are a natural modification as both models incorporate significant columns of gas. Allowing the ionisation parameter of that zone to be free in addition to the column did not further improve the fits, so we did not allow $\xi$ as a free parameter. The degeneracy between $N_{\mathrm{H}}$ and $\xi$ means that while we have modeled these residual variations as column density variations as tabulated, it is impossible to determine how much of this change is actually due to column changes and how much is due to ionisation changes.

An absorber in the line-of-sight would respond to changes in the continuum flux with recombination timescales down to seconds for gas in the density regime likely for layers close to the nucleus, and even faster ionisation timescales. In the scattering model our absorption happens after scattering, so there may be significant time lags and we would not necessarily expect to see $\xi$ changes consistent with the continuum variation. In the pcov absorption model the gas might be expected to respond to the continuum variability but it is not possible to unambiguously determine how much of the continuum variability is due to the covering variations and how much to intrinsic continuum flux changes. Given these ambiguities we do not seek consistency between observed flux changes and the ionisation state of the absorbers.

After freeing the column density for $N_{\mathrm{H}}(1)$ the fits were rerun with the column density of $N_{\mathrm{H}}(1)$ allowed to float in addition to the free parameters already noted. Freeing this column improved both models: the scattering model was improved to an overall goodness-of-fit $\chi^{2}=4274$ for 3724 d.o.f. while the $p$-cov absorption model yielded 3488 for 3724 d.o.f. for the $X M M$ data. The improvement to the fits in both cases indicates that column density changes are a significant component of spectral variability in Mrk 766 in either the scattering or absorption scenarios. While the absorption model provides a fit that appears statistically superior with current model constructions we note that the scattering model has been constructed using basic model components to attempt to describe an interesting physical scenario. The recourse to this approximation for the combined scattering and self-absorption effects that may occur in (for example) a disk wind, is due to the lack of a full self-consistent wind model at this time. For this reason, the simple fit statistic should not be a basis for ruling out this crudely-parameterized but interesting physical possibility.

\subsection{Suzaku results}

Extending our consideration to the Suzaku data we first created a light curve from the PIN data, and compared it to light curves in the $0.5-2$ and 2-10 keV bands of XIS0. Figure 4 shows those time series and it appears that while the XIS data vary by a factor $\sim 6$ from trough to peak, the PIN data are inconsistent with a zero-lag correlation in variability. However, there are some uncertainties in the production of the PIN light curve that relate to the difficulty in producing an accurate subtraction of the instrumental background level: for this reason the PIN curve is not fitted to the model predicted rates, although these are illustrated in Fig. 4.

When the PIN data are binned into the four time intervals chosen for spectral analysis the light curve is consistent with a constant flux and so we used the mean PIN spectrum to obtain a fit with each XIS time slice (Tables A.1, A.2; Figs. 5, 6).

Fitting the spectral data we found that three of four time slices were well explained in the same way as the XMM data (Tables A.1, A.2). However, the third time slice was a very poor fit to both models (reduced $\chi_{r}^{2}=1.88$ for the scattering model, $\chi_{r}^{2}=2.99$ for the p-cov absorption model) until the second large gas column, $N_{\mathrm{H}}(2)$, was also allowed to float. Allowing a second layer of absorption to vary yielded significantly better fits to both models (Tables A.1, A.2) although yet more complexity is apparently indicated (Figs. 5, 6). 

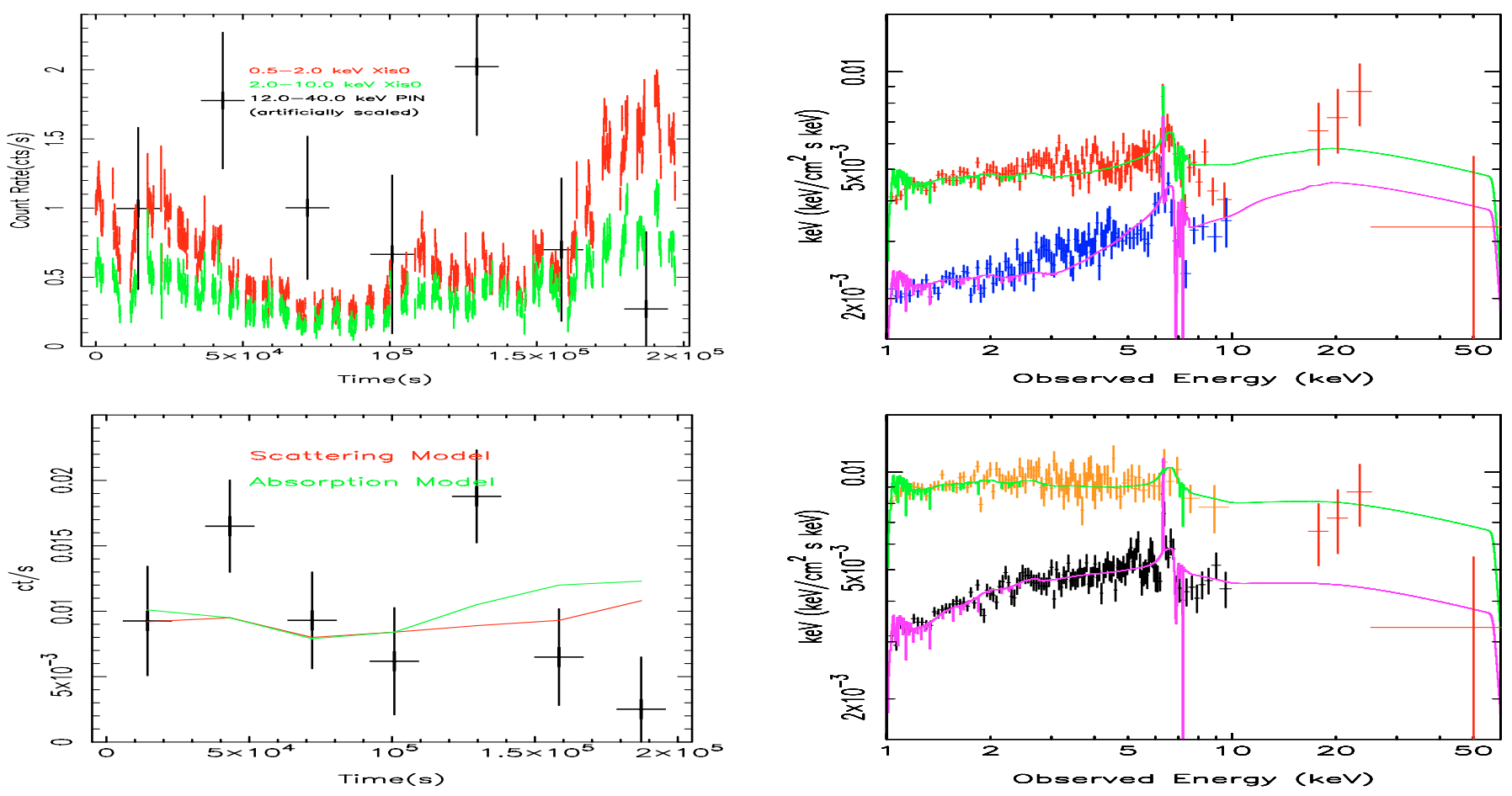

Fig. 4. Top: XIS0 time series in $500 \mathrm{~s}$ bins in the $0.5-2.0$ (red) and $2-10 \mathrm{keV}$ (green) bands and from the PIN data covering $\sim 12-40 \mathrm{keV}$ (black). Bottom: PIN light curve versus predicted flux in the $12-40 \mathrm{keV}$ band from the scattering (red) and absorption (green) models.
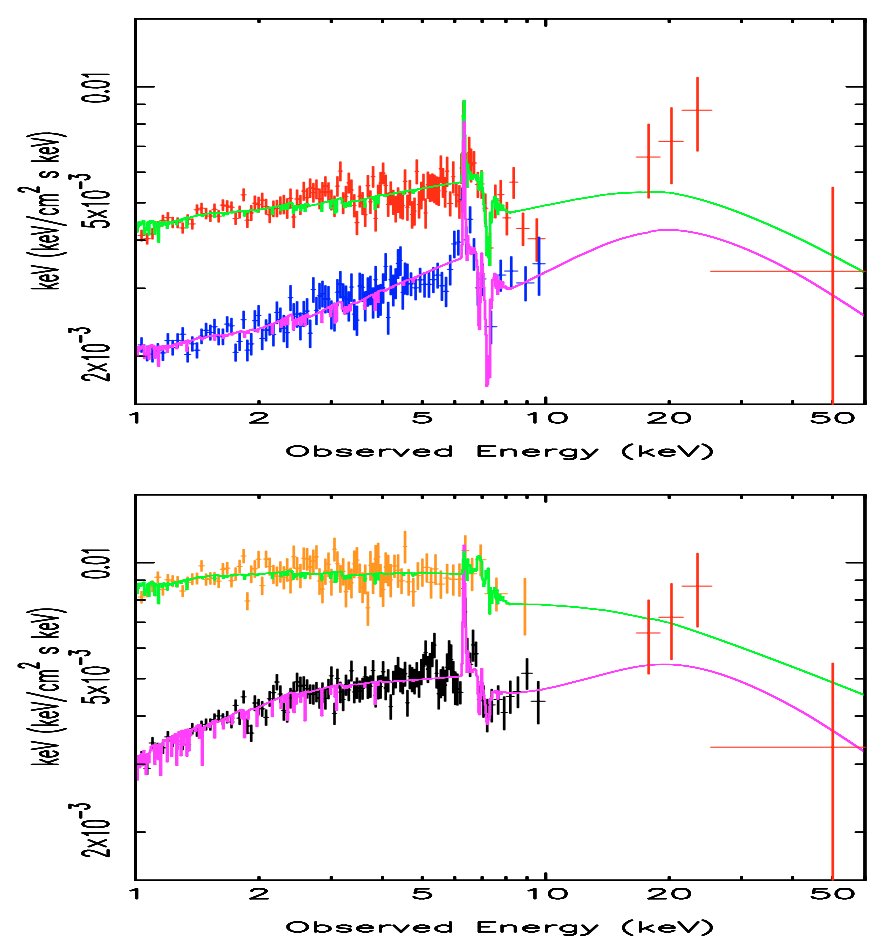

Fig. 5. Scattering model fits to the individual time slices of Suzaku data. Top: red is slice 1 , blue is slice 2. Bottom: black is slice 3, Orange is slice 4 . PIN data have been adjusted downwards by the $13 \%$ flux correction determined from simultaneous fitting of XIS and PIN spectra.

Both physical interpretations of the PCA results provide reasonable fits to all XMM spectra. Tables A.1 and A.2 show the fit parameters for each model, along with the goodness of fit

Fig. 6. P-cov absorption model fits to some of the individual time slices of Suzaku data, color coding and PIN data as for Fig. 5.

for each slice. Figure 7 shows the primary parameters for the fitted models, i.e. the flux of the two dominant spectral components. Comparison of Tables A. 1 and A. 2 shows that the p-cov absorption model provides the statistically superior fit to most time slices sampled from $X M M$ while the scattering model is the best fit to Suzaku data. However, the aim here is to determine whether scattering/reflection or absorption effects dominate the spectral variability and, as noted above, if our template models are not perfect representations of the physical scenarios then we might be misled by a simple comparison of the $\chi^{2}$ statistic. To understand the nature of the source variations we need to consider which of the sets of results makes the most physical sense, and part of this involves consideration of whether any obvious parameter correlations emerge.

\subsection{Line variability}

Figure 8 shows the absorption line fluxes plotted against time. The $6.97 \mathrm{keV}$ line appears to show significant variability while the $7.3 \mathrm{keV}$ line shows less indication of any variation; to quantify this we performed a $\chi^{2}$ test against a model representing a constant line flux. The fitted values of line flux were slightly different for the two model applications because the line depths are critically dependent on the placement of the continuum.

In the scattering model, the $6.97 \mathrm{keV}$ line flux variations yielded $\chi^{2}=66 / 31$ d.o.f, variable at significance level $\sim 2 \times 10^{-4}$, the $7.3 \mathrm{keV}$ line flux variations gave $\chi^{2}=28 / 31$ d.o.f., consistent with a constant value. In the p-cov absorption model, the $6.97 \mathrm{keV}$ line yielded $\chi^{2}=71 / 31$ d.o.f, also variable at significance level $\sim 5 \times 10^{-5}$, but the $7.3 \mathrm{keV}$ line gave $\chi^{2}=52 / 31$ d.o.f., variable at significance level 0.01 . In this case the evidence for variation in the $7.3 \mathrm{keV}$ line turns out to be due to one deep line measurement during the low state in spectral slice 9 , exclusion of 

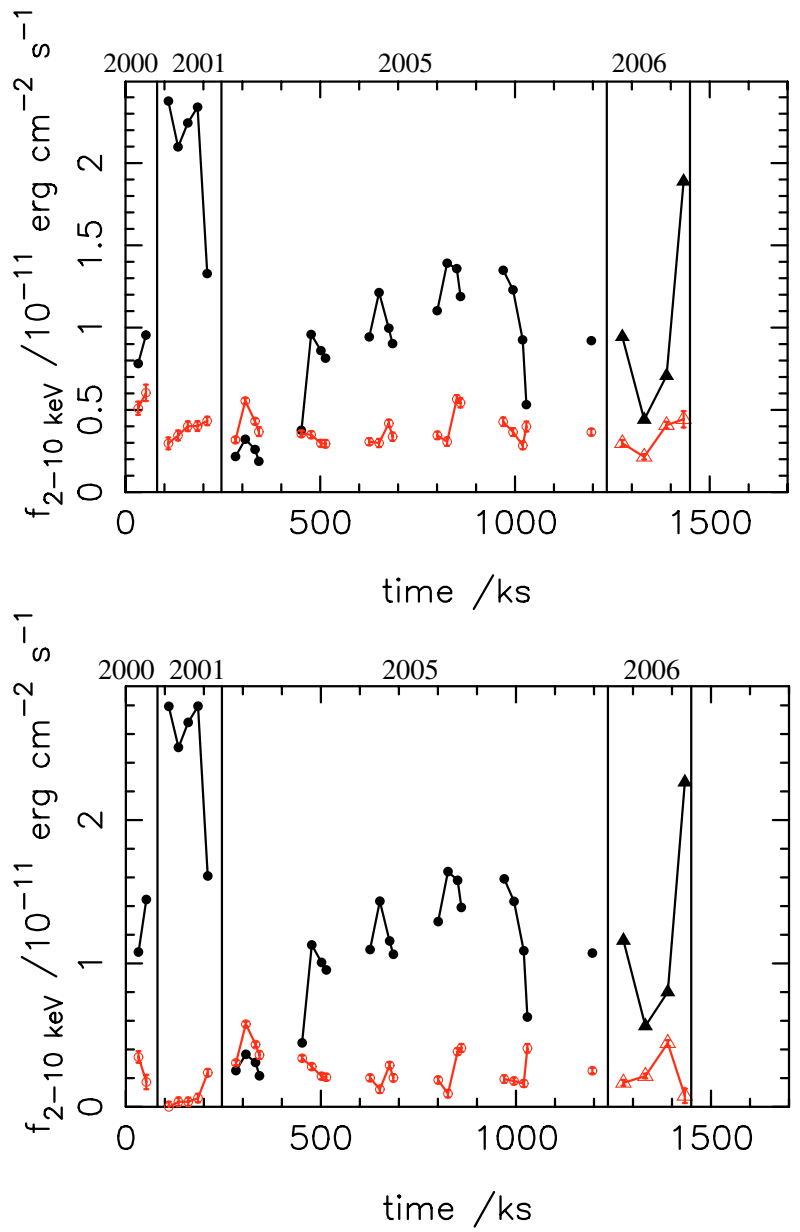

Fig. 7. Top: direct (black, solid symbols) and scattered (red, open symbols) component amplitudes of the source flux for the scattering model. Bottom: direct (black, solid symbols) and absorbed (red, open symbols) component amplitudes of the source flux from the partial covering model. Solid vertical lines show where long breaks in time have been omitted for clarity.

that single value shows the rest of the line fluxes to be consistent with a constant value.

In spite of the dependence of the lines on the continuum model, the absorption line at $6.97 \mathrm{keV}$ shows significant variability in flux for both of the different parameterizations of the data. This strengthens our confidence in the robustness of the line variability result in this case. However the discrepant conclusions on the $7.3 \mathrm{keV}$ absorption line in the two scenarios leads us to conclude we cannot be sure of the variability behavior of this line component. However, the equivalent width of the $7.3 \mathrm{keV}$ line does vary systematically with the total source flux and is not consistent with being a line of constant equivalent width.

The emission line at $6.4 \mathrm{keV}$ also has different implied behavior in the scattering and p-cov absorption models. In the former it is tied to the neutral reflector and measures of the line flux yield $\chi^{2}=70 / 31$ d.o.f (variable at significance level $8 \times 10^{-5}$ ) while in the p-cov absorption model it is not linked to any other continuum component and yields $\chi^{2}=8.3 / 31$ d.o.f, consistent with a constant value. The question of variability of this line component remains ambiguous, while the Fe emission varies in this regime it is impossible to disentangle the neutral line from the wings of the emission line from the ionized reflector and the origin of the neutral line cannot be conclusively determined.
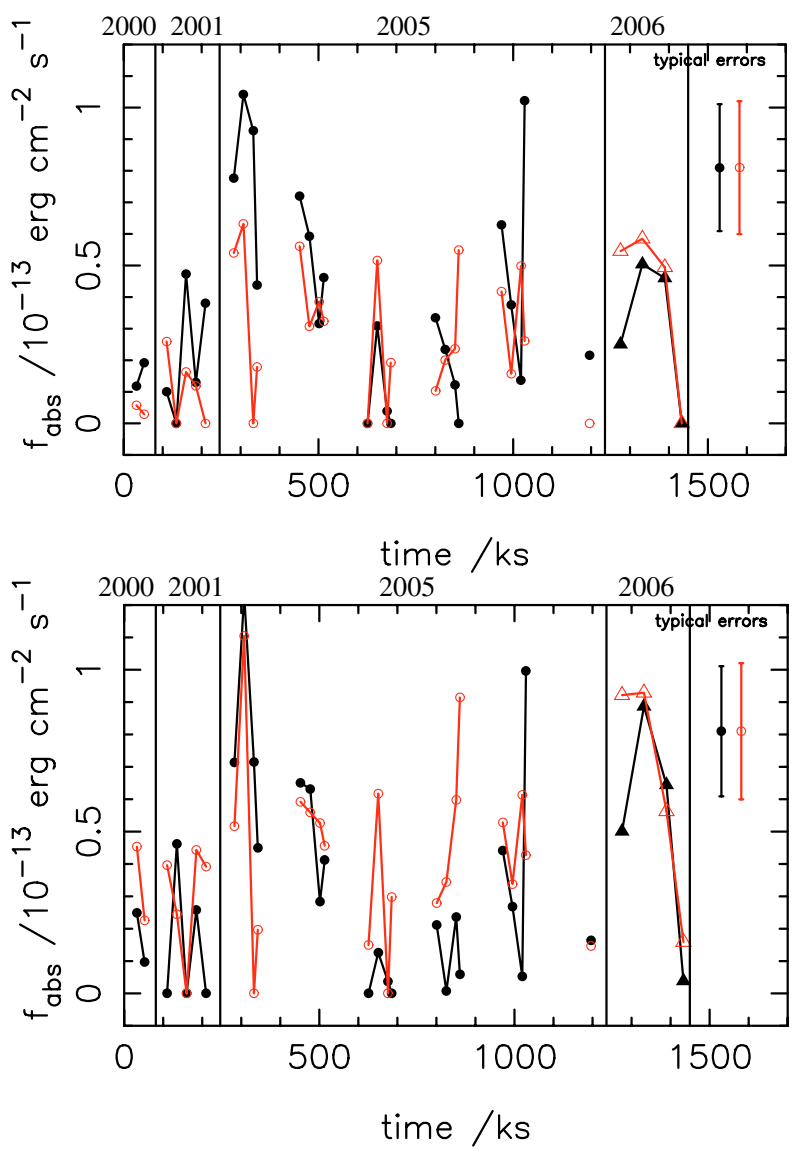

Fig. 8. Absorption line fluxes for lines at $7.3 \mathrm{keV}$ (red, open symbols) and $6.97 \mathrm{keV}$ (black, solid symbols) from the scattering (top) and partial-covering (bottom) fits.

\section{Parameter correlations}

The presence or lack of compelling correlations between parameters could help us distinguish between the scattering and partial-covering models. The achievement of reasonable fits (Tables A.1, A.2) means reliable parameters can now be extracted and we can investigate the correlations between them for each of the models.

First we plot the most robustly-measured parameters, the directly-viewed and scattered or absorbed fluxes. Figure 7 shows the directly viewed flux (powerlaw plus correlated ionized reflector) for both models (black line) compared to (red) the scattered fraction (top panel) and absorbed fraction (bottom panel). The flux history of the source reveals how the 2000-2001 epochs were dominated by a direct view of the power-law continuum and associated ionized reflector. The 2005 observation found Mrk 766 at a very low flux rising later in the observation. In 2006 we again found the source in a fairly low state but with a rapid rise evident towards the end of the Suzaku observation.

Overall the steady level of the scattered flux in Fig. 6 is consistent with the PCA, that analysis indicated the scattered component to vary within bounds $\pm 38 \%$ of the mean, although the component is higher than expected at the start of the 2000 observations. In the partial-covering model, some anti-correlation of directly-viewed and absorbed fractions is a consequence of the model construction. 
Table 1. One-tailed significance level of correlations between parameters in the scattering model (upper right quadrant) and p-cov absorption model (lower-left quadrant) fitted to XMM and Suzaku data and after allowing column variability for $N_{\mathrm{H}}(1)$. Anticorrelations are indicated by minus signs in front of each value of significance level.

\begin{tabular}{lcccc}
\hline \hline & Direct & Hard & $6.97 \mathrm{keV}$ & $7.3 \mathrm{keV}$ \\
\hline direct & - & 0.68 & -0.0013 & -0.033 \\
hard & $-7 \times 10^{-7}$ & - & 0.50 & -0.05 \\
$6.97 \mathrm{keV}$ & $-9 \times 10^{-5}$ & 0.0010 & - & 0.0061 \\
$7.3 \mathrm{keV}$ & -0.1447 & 0.0949 & 0.0071 & - \\
\hline
\end{tabular}

\subsection{Absorption line correlations}

Next we investigate the relationship between the absorbed or scattered fraction, and the narrow absorption lines. The strength of the correlations between modeled quantities is assessed using Spearmann's rank correlation coefficient, assuming data points to be statistically independent, along with correlation parameters for all other combinations of variables in both models. The red-noise power-spectrum (see Miller et al. 2007) of the intrinsic source variations causes some degree of correlation between the modeled quantities, so the significance of correlations may be overestimated by this approach, but without more information on the power-spectrum of variations in each quantity it is hard to correct for this effect. In fact, the statistical uncertainties on the line flux values are sufficiently large that this red-noise effect has only a limited influence, although we should bear in mind that the effect may be present at some level and we should use the significance values as being a relative measure of which relationships are the most important rather than being an absolute measurement of significance.

Considering first the scattering model results (Table 1, upper right quadrant), the flux of the $6.97 \mathrm{keV}$ absorption line appears to be anti- correlated with the directly-viewed component and not correlated with the quasi-constant scattered component (Figs. 9, 10).

In the p-cov absorption model parameterization the $6.97 \mathrm{keV}$ absorption line is anti-correlated with the direct flux or correlated with the absorbed flux, with a slight preference for the former scenario (Figs. 9, 10). As the uncovered and covered fractions are anti-correlated in this model, it is hard to discern which has the primary correlation with the absorption line.

The $7.3 \mathrm{keV}$ absorption line flux does not show a correlation with anything in either model (Figs. 9, 10). However, the line equivalent width is not consistent with being constant when measured against the varying spectral component, but is consistent with a constant value against the scattered or absorbed components in the two models.

\section{Discussion}

The ambiguity in interpretation of the hard X-ray spectra observed in the low flux state for many Seyfert galaxies has hindered progress understanding these sources. We have attempted to move forward by using a large dataset accumulated by XMM over 2000-2005, combined with data from the broad bandpass covered by Suzaku during a 2006 observation, for the highly X-ray variable narrow-line Seyfert 1, Mrk 766.

Two models have been directly compared in application to time-selected spectra from Mrk 766. The aim of the comparison has been to distinguish whether large-scale spectral variability is best explained by changes in relative contribution from powerlaw plus ionized-reflector compared to the scattering
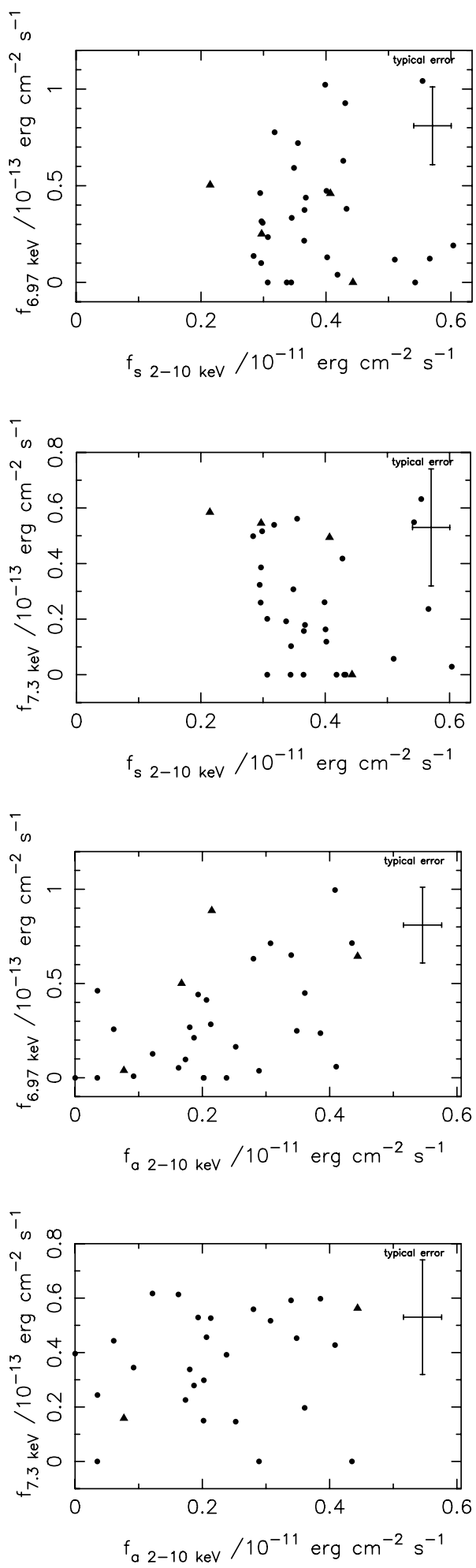

Fig. 9. Absorption line fluxes versus the hard component in the scattering model (top two panels) and p-cov model (bottom two panels) for $X M M$ (dots) and Suzaku (triangles) data.

component, or by variations in the covering fraction of the absorber. It is very important to determine the dominant effects as several models that fit the mean spectra of Seyfert galaxies comparably well have very different physical interpretations.

The most robust measurements are those of the directly viewed and scattered or absorbed fractions of flux. The obvious interpretations outlined by Miller et al. (2007) are briefly 

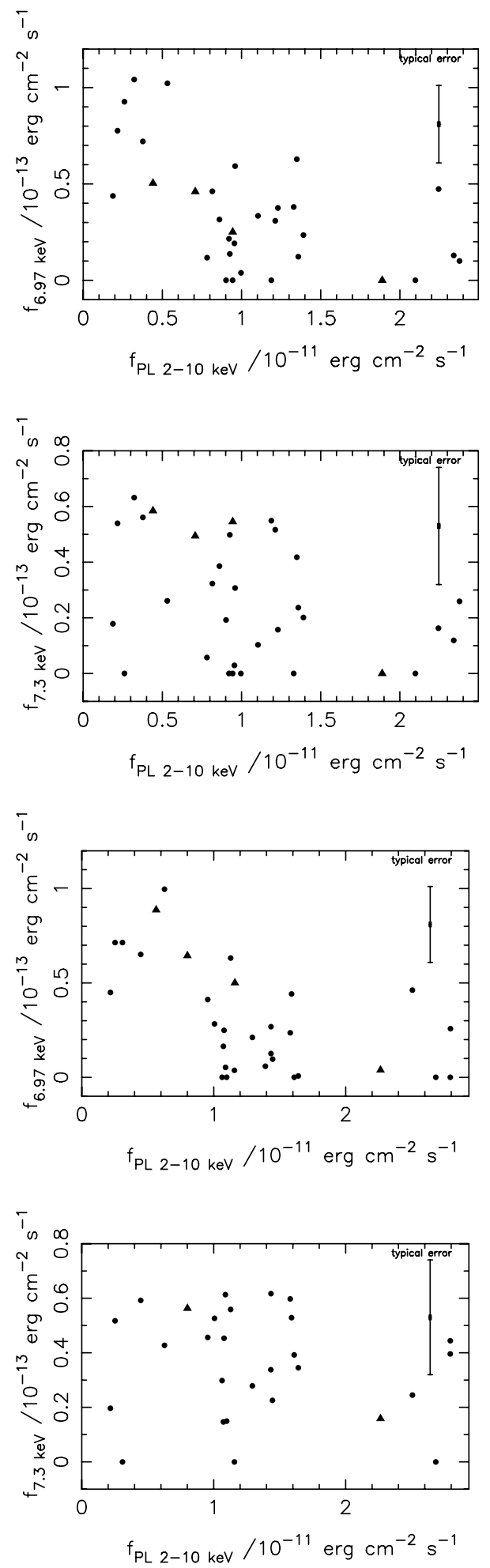

Fig. 10. Absorption line fluxes versus the direct/unabsorbed component for the scattering model (top two panels) and p-cov model (bottom two panels) for XMM (dots) and Suzaku (triangles) data.

reviewed here to set the scene for further discussion. In the scattering model the hard emission component is steady, explained by scattering from an extended region such as a disk wind that could also absorb scattered photons. PCA limits the flux variations in this component to $\pm 38 \%$ of the mean and this relatively low level of variability implies a size of at least several light days for this gas but with an indication of some response of the inner region of the wind to changes in continuum flux.

The most likely candidate for the scattering gas is the highlyionized inner zone that produces the $6.97 \mathrm{keV}$ absorption line. The scattered fraction of emission is a significant fraction of the total and so this zone likely has a large covering fraction, whereas the lower ionization layers further out probably have too small a covering to produce a significant flux of scattered X-rays. Opacity changes observed in the data could be explained by variations in such a wind that affect the degree of self-absorption of the scattered photons.

Seyfert galaxies generally (e.g. Vaughan \& Fabian 2004; Pounds et al. 2004) show the behavior exhibited by Mrk 766, i.e. a hard spectral form when the primary continuum flux is faint; this tells us something about spectral variability in the context of absorption models. The simple scenario of an intrinsically variable continuum with additional variability caused by covering fraction changes from passage of clouds across the sightline can be ruled out. The covering fraction would have to know about the continuum flux for the observed phenomena to be so widely seen, else we would see some highly absorbed high-state spectra both in general and also specifically within the long duration Mrk 766 observations: the observed variability would not be a natural consequence of such a "mixed variability" picture. Two variants of the p-cov absorption model seem plausible however, either the continuum drives apparent absorption changes by ionizing some of the gas (see later) or the continuum is intrinsically constant and the observed variability simply a consequence of the changes in covering fraction. The $\mathrm{p}$-cov absorption model fits the $X M M$ data very well but is a less satisfactory fit to the Suzaku data; as the absorption model was built upon a PCA deconstruction of the XMM data, changes in the absorbing gas between those observations and the Suzaku epoch would naturally result in a poor fit to the latter. The more we free the column and ionization-states of the layers, the better the Suzaku data are fitted, so in conclusion, the statistical quality of fits to Suzaku spectra could be interpreted as evidence for absorption changes on year-timescales: these must be expected, when opacity changes on tens of ks are also evident in the data.

One compelling alternative interpretation of the partialcovering behavior in the absorption model may be that the region producing the hard X-ray continuum might vary in extent. In this alternative picture, when the putative coronal region producing X-rays is largest the continuum could be visible above gas structures that might normally hide the nucleus: this would give apparent changes in covering fraction as the comptonizing region expands and contracts. This could naturally yield a constant flux of absorbed continuum emission that is obscured and that dominates above $10 \mathrm{keV}$. In this case the correlated variations in ionized reflection and power law continuum known in Mrk 766 (Miller et al. 2007) could be attributed to the reflector seeing more continuum photons when the coronal region is at its largest. However, perhaps a more standard interpretation of the "p-cov absorption" picture would be to suppose that the absorbing material is an inhomogeneous disk wind, in which clumps of obscuring material pass between a central continuum source and the observer. A wind would naturally have the most ionized zones closest to the nucleus, consistent with these data.

Considering the second-order spectral variability (not addressed in the principal components analysis of Miller et al. 2007): we find opacity variations in one of the absorption layers can account for most of the observed second-order effects. Degeneracy between column density and ionisation parameter means we cannot isolate the origin of the opacity changes with 
current data but again, the opacity variations support the general picture of an important disk wind component, driving the observed spectral variability.

It is interesting to note that both models presented here are broadly consistent with the soft-band data down to $0.5 \mathrm{keV}$ Fig. 3. The partial-covering absorption model provides a particularly good fit to the scattering model, and naturally explains the soft-band spectral curvature without recourse to any separate "soft excess" component. The agreement between data and model illustrates the applicability of either model over the broad range of flux covered by the source during the XMM observations. The model lines are shown extrapolated down to $0.5 \mathrm{keV}$ illustrating how exclusion of the softest data from the fits has not compromised our ability to distinguish models; the soft data indicate a preference for the partial-covering absorption model

One important diagnostic result is that the absorption line at $6.97 \mathrm{keV}$, discovered in the mean spectrum (Miller et al. 2007) shows significant variability, correlated with other aspects of source behavior. Interestingly the absorption line itself is not modeled within any of the zones of gas used to describe the overall spectral curvature but, as evident in Tables A.1, A.2, the opacity changes themselves are anti-correlated with the flux of the direct component. All zones of gas may be responding to continuum changes, or continuum changes themselves may be an artifact of variable absorption. This possibility has been considered previously: the tight correlation between the ionized reflector and continuum flux might be a consequence of variable occultation of both if Compton-thick "bricks" of variable covering fraction occult continuum and reprocessed emission from within. The bricks would be implied to exist $\sim 70 r_{\mathrm{g}}$ (Miller et al. 2007) which is close to where the occulted line is estimated to arise (based on the emission line width). By similar arguments, opacity changes observed on comparable timescales driven by motion of clouds across the sight-line would indicate clouds at the same radial location. If the opacity changes originate as ionisation-state variations then the location is currently unconstrained.

The existence of a strong isolated absorption line at $6.97 \mathrm{keV}$ yields the lower $\operatorname{limit} \log \xi \gtrsim 4$, i.e. it comes from gas that is the most highly-ionized of all layers evident in the $2-10 \mathrm{keV}$ band. It is clear that the other layers of lower $\xi$ gas may exist along the same sight-line but at larger radial distances from the central source: the highest- $\xi$ zone could not exist outside the other gas layers as shielding would prevent it achieving $\log \xi \gtrsim 4$. Similar models have been discussed for sources such as e.g., NGC 3516 (Turner et al. 2005) and NGC 4151 (Kraemer et al. 2005) and thus it seems that spectral variations driven by a time-variable disk wind may be applicable across the Seyfert population.

The $6.97 \mathrm{keV}$ absorption line is so strong that it is not possible to model the zone from which it originates without invoking a significant turbulent velocity for the absorbing gas, again, consistent with its origin very close to the active nucleus. Low turbulent velocities (few hundred $\mathrm{km} \mathrm{s}^{-1}$ ) predict much weaker lines even for columns densities of few $\times 10^{24} \mathrm{~cm}^{-2}$ as the line saturates quickly and the equivalent width predicted when line broadening occurs can be much higher. One must invoke a turbulent velocity $\gtrsim 2000 \mathrm{~km} \mathrm{~s}^{-1}$ to fit the observed line at its deepest with a column that is Compton-thin; for a turbulent velocity of $3000 \mathrm{~km} \mathrm{~s}^{-1}$ we find a fit for the line in the mean spectrum using a column of gas with $N_{\mathrm{H}} \sim 5 \times 10^{23} \mathrm{~cm}^{-2}$. Variability in the absorption line at $6.97 \mathrm{keV}$ supports the general conclusion that rapid absorption variation is very important: the presence of such a line strengthens previous suggestions (Nandra \& Pounds 1994; Turner et al. 2005; Reeves et al. 2004) that very large column densities of highly-ionized gas exist close to the nucleus, dangerously close to the conditions that cause most confusion with Fe emission features.

The absorption-line correlations found here seem to present two possibilities: in the p-cov absorption model: the $6.97 \mathrm{keV}$ line flux variations could be due either to being constant EW absorption in front of the fluctuating absorbed part, or to being over-ionized at times of exposure to high powerlaw flux. Figure 9 shows that the $6.97 \mathrm{keV}$ line flux does indeed correlate positively with the flux in the absorbed component, lending weight to the first of these possibilities. There is however a stronger anticorrelation with the direct component (Fig. 10, Table 1), which appears to show a sudden transition in line flux for direct component flux $f_{2-10 \mathrm{keV}} \gtrsim 10^{-11} \mathrm{erg} \mathrm{cm}^{-2} \mathrm{~s}^{-1}$. We discuss below the difficulty of obtaining such a rapid transition purely from changing ionization parameter.

Compared with the $6.97 \mathrm{keV}$ line variations, the $7.3 \mathrm{keV}$ line behavior is less clear due to its model-dependency. One thing that is clear is that the feature is persistent, remaining evident in Suzaku data during 2006 and that it is not an artifact of any instrument. However, the identification of this line is unknown, there are no known transitions expected of this strength at such a rest-energy without a substantial complex of other lines appearing (Kallman et al. 2004). It is possible that this feature represents an ionized Fe edge whose shape is distorted due to unresolved features close in energy, or as noted by Miller et al. (2007) the feature could arise in a high-velocity outflow such as from Fe XXVI Ly $\alpha$ in gas with a bulk outflow velocity $\sim 13000 \mathrm{~km} \mathrm{~s}^{-1}$. There is a suggestion that the $7.3 \mathrm{keV}$ line variations are correlated with those of the $6.97 \mathrm{keV}$ line, however, there is no evidence for a causal relation between the $7.3 \mathrm{keV}$ line and the direct component in the p-cov absorption model, and only weak evidence for correlation with the direct component in the scattering model (Table 1). This lack of correlation is, in itself interesting. The equivalent width of the $7.3 \mathrm{keV}$ line is inconsistent with a constant value when measured against the variable spectral component in either model, but is consistent with a constant value when measured against the hard/steady component in either model: this may be associated with a quasiconstant continuum component, leaving the equivalent width variations attributed to changes in the flux of the direct continuum component.

In the scattering model, the variation in $6.97 \mathrm{keV}$ line flux would need to be explained as arising from a change in ionization state of the absorbing gas as the source brightens overall. It appears that the decrease in line flux is associated with quite a sharp transition in total source brightness which would not be expected given the broad range of ionization parameter over which any given ionization state generally exists (Kallman et al. 2004). We can test this explanation by generating model absorbers with XSTAR assuming that the line is Fe XXVI $6.97 \mathrm{keV}$ and assuming that the ionization parameter varies with the total source brightness. As discussed above, the apparent lack of Fe K $\alpha 6.7 \mathrm{keV}$ in absorption indicates high ionization, $\log \xi>4$. As also discussed above, in order to obtain a high equivalent width without an extreme column density some velocity broadening is probably required, and we test models with velocity broadening $\sigma=3000 \mathrm{~km} \mathrm{~s}^{-1}$, a value that allows reconciliation of the measured line strength with physical models, while staying within the velocity constraint yielded from the data $\left(\sigma<6000 \mathrm{~km} \mathrm{~s}^{-1}\right)$. The results are shown in Fig. 11, where the equivalent width has been measured against the total continuum flux: i.e. it has been assumed that the absorbing gas lies in front of all the source emission components. The radial distance and density of the 


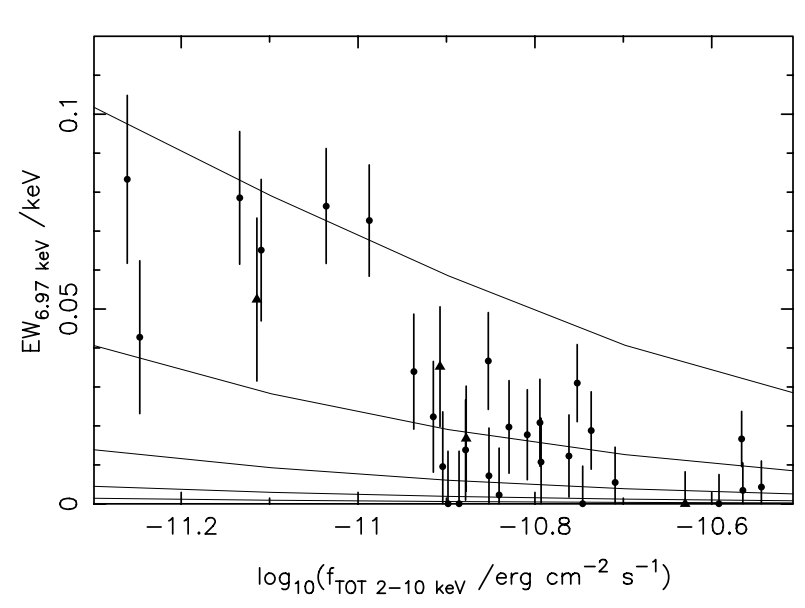

Fig. 11. Variation of equivalent width of the $6.97 \mathrm{keV}$ line, measured against the total source continuum, with the total source flux (points with error bars: for XMM (dots) and Suzaku (traingles) data). Values are calculated within the scattering model. Also shown are model lines for the variation expected from XSTAR models of the Fe XXVI $6.97 \mathrm{keV}$ line arising from ionisation variation alone, for column densities with solar abundance of $N_{\mathrm{H}}=10^{21.5}, 10^{22}, 10^{22.5}, 10^{23}, 10^{23.5} \mathrm{~cm}^{-2}$.

absorbing material is unknown, so we have investigated the cases in the range $n=10^{8}-10^{12} \mathrm{~cm}^{-3}$ and choose to adjust the ionization parameter so that the maximum possible variation in line equivalent width is produced, corresponding to $\log \xi \sim 5$.

It appears that indeed the rapid variation in equivalent width cannot be well reproduced simply by changes in source brightness affecting the ionization state of the absorber. It may be, however, that the particular absorbing zone lies only in front of the scattered component of emission, but that nonetheless its ionization state is still determined by the total source brightness. In this case the typical equivalent width measured against the scattered component is higher, but shows less variation. Although the ionization-variation hypothesis still does not produce good agreement with the observed variation, such a picture probably cannot be ruled out. This deduction does have an important implication for the nature of the scattering component. If the $6.97 \mathrm{keV}$ absorbing gas has to be associated with this hard spectral component only, then that component cannot be significantly relativistically blurred, otherwise the absorption line would also be blurred. But if the absorbing gas lies outside any such blurred region, the rapid variation in $\mathrm{EW}$ with source flux would not take place. This consideration seems to rule out relativistically blurred models for the scattered component, unless an alternative blurred model can be found that reduces the significance of the $6.97 \mathrm{keV}$ absorption line.

\section{Conclusions}

Detailed time-resolved X-ray spectroscopy has confirmed that the spectral shape of Mrk 766 is directly coupled to the source brightness. Two model interpretations of principal components analysis fit the time-sliced data well with changes in spectral shape then attributed to the relative strength of the continuum and a self-absorbed scattered component, or to changes in covering fraction of an absorber. In addition to this dominant mode of variation, significant temporal variations in opacity down to timescales of tens of ks are also required to fit the timesliced data as previously indicated from residuals in the principal components analysis (Miller et al. 2007). The previouslyreported absorption line at $6.97 \mathrm{keV}$ is likely Fe XXVI K $\alpha$ from gas with $\log \xi \gtrsim 4$ and this varies correlated with the primary continuum flux, tracing the behavior of a highly-ionized gas zone and extending our understanding of the parameter-space spanned by the complex, multi-layered absorber in this Seyfert galaxy.

In conclusion then, Mrk 766 shows strong evidence for stratified layers of absorption, possibly decreasing in ionization with increasing radial location. The gas layers have rapidly variable covering factor, opacity and ionization state and are required to explain the spectral variability in this source whether or not one allows the lowest flux state to be dominated by some residual scattered continuum component. Some scattered and reflected emission must be present to explain the observations, especially the rapidly variable emission features that correlate with continuum flux, most evident in the high state. These results, combined with evidence in the literature for significant bulk outflow velocities in X-ray absorbers in general (Crenshaw et al. 2003) point to disk winds as dominant in shaping Seyfert spectral variations in the X-ray band.

Acknowledgements. We thank Brian Wingert for help with the Suzaku data reduction. We thank the referee, Chris Done, for comments that helped us clarify and otherwise improve the paper.

\section{References}

Arnaud, K. 1996, Astronomical Data Analysis Software and Systems V, ed. G. H. Jacoby, \& J. Barnes, ASP Conf. Ser., 101, 17

Boldt, E., \& Leiter, D. 1987, ApJ, 322, 1

Crenshaw, D. M., Kraemer, S. B., \& George, I. M. 2003, ARA\&A, 41, 117

George, I. M., \& Fabian, A. C. 1991, MNRAS, 249, 352

Gruber, D. E., Matteson, J. L., Peterson, L. E., \& Jung, G. V. 1999, ApJ, 520, 124

Guilbert, P. W., \& Rees, M. J. 1988, MNRAS, 233, 475

Kallman, T., Palmeri, P., Bautista, M. A., Mendoza, C., \& Krolik, J. H. 2004, ApJS, 155, 675

Kraemer, S. B., George, I. M., Grenshaw, D. M., et al. 2005, ApJ, 633, 693

Laor, A. 1991, ApJ, 376, 90

Lightman, A. P., \& White, T. R. 1988, ApJ, 335, 57

Miller, L., Turner, T. J., Reeves, J. N., et al. 2006, A\&A, 453, L13

Miller, L., Turner, T. J., Reeves, J. N., et al. 2007, A\&A, 463, 131

Nandra, K., \& Pounds, K. A. 1994, MNRAS, 268, 405

Nandra, K., George, I. M., Mushotzky, R. F., Turner, T. J., \& Yaqoob, T. 1997, ApJ, 477, 602

Ogle, P. M., Brookings, T., Canizare, C. R., Lee, J. C., Marshall, H. L. 2003, A\&A, 402, 849

Osterbrock, D. E., \& Pogge, R. W. 1985, ApJ, 297, 166

Perola, G. C., Matt, G., Cappi, M., et al. 2002, A\&A, 389, 802

Pounds, K. A., Reeves, J. N., Page, K. L., \& O’Brien, P. T. 2004, ApJ, 605, 670

Reeves, J. N., Nandra, K., George, I. M., et al. 2004, ApJ, 602, 648

Ross, R. R., \& Fabian, A. C. 2005, MNRAS, 358, 211

Strüder, L., Briel, U., Dennerl, K., et al. 2001, A\&A, 365, L18

Tanaka, Y., Nandra, K., Fabian, A., et al. 1995, Nature, 375, 659

Turner, T. J., Kraemer, S .B., George, I. M., Reeves, J. N., \& Bottorff, M. C. 2005, ApJ, 618, 155

Turner, T. J., Miller, L., George, I. M., \& Reeves, J. N. 2006, A\&A, 445, 59

Vaughan, S., \& Fabian, A. C. 2004, MNRAS, 348, 1415

Zdziarski, A. A., Johnson, W. N., Done, C., Smith, D., \& McNaron-Brown, K. 1995, ApJ, 438, L63 
T. J. Turner et al.: Time-resolved spectroscopy of Mrk 766, Online Material p 1

\section{Online Material}




\section{Appendix A: Model fit parameters}

A.1 and A.2 give the model fit parameters for the scattering and absorption models applied to the $X M M$ and Suzaku data. The model components are described in the main body of the paper. The values given in each time slice (column one) are: the $2-10 \mathrm{keV}$ fluxes of the total, direct and scattered or absorbed components (Cols. 2-4); the absorption-line flux in each of the $6.97 \mathrm{keV}$ and $7.3 \mathrm{keV}$ (rest-frame) absorption features (Cols. 5, 6); the emission-line flux in the $6.4 \mathrm{keV}$ emission line (Col. 7); the $N_{\mathrm{H}}$ column density (Col. 8); and the reduced $\chi_{r}^{2}$, noting that the $X M M$ and Suzaku fits have differing degrees of freedom. 
T. J. Turner et al.: Time-resolved spectroscopy of Mrk 766, Online Material p 3

Table A.1. Values of fitted parameters for the "scattering" model, in each time slice, as described in the text.

\begin{tabular}{|c|c|c|c|c|c|c|c|c|}
\hline \multicolumn{9}{|c|}{ Scattering model } \\
\hline $\begin{array}{l}\text { Time } \\
\text { Slice }\end{array}$ & $\begin{array}{l}\text { Total } \\
2-10\end{array}$ & $\begin{array}{c}\text { Direct } \\
\mathrm{keV} \text { flux } / 10^{-11}\end{array}$ & $\begin{array}{r}\text { Scattered } \\
\text { erg cm} \text { cm }^{-2} \mathrm{~s}^{-1}\end{array}$ & $\begin{array}{l}6.97 \mathrm{keV} \\
\text { Line pho }\end{array}$ & $\begin{array}{l}7.30 \mathrm{keV} \\
\mathrm{n} \text { flux } / 10^{-6}\end{array}$ & $\begin{array}{l}6.4 \mathrm{keV} \\
\mathrm{cm}^{-2} \mathrm{~s}^{-1}\end{array}$ & $\begin{array}{c}N_{\mathrm{H}}(1) \\
/ 10^{22} \mathrm{~cm}^{-2}\end{array}$ & $\chi^{21}$ \\
\hline \multicolumn{9}{|c|}{$2000 X M M$} \\
\hline 1 & 1.406 & $0.782 \pm 0.010$ & $0.510 \pm 0.040$ & $1.1 \pm 2.0$ & $0.5 \pm 1.9$ & $9.4 \pm 1.0$ & $0.8_{-0.1}^{+0.2}$ & 1.00 \\
\hline \multicolumn{9}{|c|}{$2001 X M M$} \\
\hline 3 & 2.724 & $2.377 \pm 0.006$ & $0.296 \pm 0.036$ & $0.9 \pm 2.9$ & $2.2 \pm 2.8$ & $4.5 \pm 3.0$ & $8.3_{-0.5}^{+0.7}$ & 1.56 \\
\hline 4 & 2.560 & $2.098 \pm 0.007$ & $0.344 \pm 0.030$ & $0.0 \pm 2.7$ & $0.0 \pm 2.7$ & $9.1 \pm 2.0$ & $3.3_{-0.7}^{+0.5}$ & 1.29 \\
\hline 5 & 2.715 & $2.244 \pm 0.007$ & $0.401 \pm 0.030$ & $4.2 \pm 2.7$ & $1.4 \pm 2.7$ & $6.4 \pm 2.0$ & $3.6_{-0.8}^{+0.7}$ & 1.28 \\
\hline 6 & 2.858 & $2.340 \pm 0.004$ & $0.402 \pm 0.030$ & $1.2 \pm 2.9$ & $1.0 \pm 2.8$ & $9.7 \pm 2.0$ & $3.6_{-0.07}^{+0.6}$ & 1.33 \\
\hline 7 & 1.835 & $1.329 \pm 0.007$ & $0.433 \pm 0.026$ & $3.4 \pm 2.5$ & $0.0 \pm 2.7$ & $6.2 \pm 2.0$ & $2.3_{-0.3}^{+0.4}$ & 1.01 \\
\hline \multicolumn{9}{|c|}{$2005 X M M$} \\
\hline 8 & 0.548 & $0.216 \pm 0.003$ & $0.318 \pm 0.017$ & $7.0 \pm 1.3$ & $4.6 \pm 1.2$ & $2.3 \pm 2.0$ & $7.9_{-0.3}^{+0.3}$ & 1.20 \\
\hline 9 & 0.920 & $0.322 \pm 0.003$ & $0.555 \pm 0.017$ & $9.3 \pm 1.6$ & $5.4 \pm 1.5$ & $5.0 \pm 2.0$ & $3.5_{-0.2}^{+0.3}$ & 1.37 \\
\hline 10 & 0.735 & $0.260 \pm 0.003$ & $0.431 \pm 0.018$ & $8.3 \pm 1.4$ & $0.0 \pm 1.6$ & $4.3 \pm 3.0$ & $5.8_{-0.2}^{+0.2}$ & 0.87 \\
\hline 11 & 0.566 & $0.187 \pm 0.004$ & $0.368 \pm 0.027$ & $3.9 \pm 2.4$ & $1.5 \pm 2.4$ & $1.5 \pm 3.0$ & $9.2_{-10}^{-0.12}$ & 1.16 \\
\hline 12 & 0.777 & $0.377 \pm 0.004$ & $0.355 \pm 0.018$ & $6.5 \pm 1.5$ & $4.8 \pm 1.4$ & $4.8 \pm 3.0$ & $4.7^{+0.3}$ & 1.12 \\
\hline 13 & 1.404 & $0.959 \pm 0.005$ & $0.349 \pm 0.021$ & $5.3 \pm 1.9$ & $2.6 \pm 1.8$ & $8.6 \pm 3.0$ & $2.4_{-0.3}^{-0.3}$ & 1.26 \\
\hline 14 & 1.217 & $0.861 \pm 0.004$ & $0.297 \pm 0.020$ & $2.8 \pm 1.8$ & $3.3 \pm 1.8$ & $5.5 \pm 3.0$ & $3.7_{-0.1}^{+0.5}$ & 1.20 \\
\hline 15 & 1.156 & $0.815 \pm 0.004$ & $0.295 \pm 0.023$ & $4.1 \pm 1.9$ & $2.8 \pm 1.8$ & $4.5 \pm 2.0$ & $4.2_{-0.06}^{+0.5}$ & 1.10 \\
\hline 16 & 1.301 & $0.944 \pm 0.005$ & $0.307 \pm 0.021$ & $0.0 \pm 1.9$ & $0.0 \pm 1.9$ & $4.1 \pm 2.0$ & $4.0_{-0.0}^{+0.5}$ & 1.38 \\
\hline 17 & 1.554 & $1.213 \pm 0.005$ & $0.299 \pm 0.025$ & $2.8 \pm 2.0$ & $4.4 \pm 1.9$ & $4.1 \pm 3.0$ & $4.9_{-0.06}^{+0.6}$ & 1.31 \\
\hline 18 & 1.444 & $0.996 \pm 0.005$ & $0.418 \pm 0.022$ & $0.4 \pm 2.1$ & $0.0 \pm 2.1$ & $2.0 \pm 3.0$ & $3.8_{-04}^{+0.6}$ & 1.10 \\
\hline 19 & 1.264 & $0.902 \pm 0.004$ & $0.337 \pm 0.025$ & $0.0 \pm 2.2$ & $1.6 \pm 2.1$ & $2.2 \pm 2.0$ & $\begin{array}{l}3.0-0.4 \\
4.9_{-0.5}^{+0: 5}\end{array}$ & 1.11 \\
\hline 20 & 1.482 & $1.104 \pm 0.004$ & $0.345 \pm 0.023$ & $3.0 \pm 2.0$ & $0.9 \pm 1.9$ & $3.1 \pm 3.0$ & $4.5^{-0.5}$ & 1.15 \\
\hline 21 & $\begin{array}{l}1.402 \\
1.732\end{array}$ & $1.391 \pm 0.004$ & $0.307 \pm 0.026$ & $2.1 \pm 2.1$ & $1.7 \pm 2.0$ & $3.2 \pm 3.0$ & $5.3^{-0.5}$ & 1.04 \\
\hline 22 & 1.953 & $1.358 \pm 0.004$ & $0.566 \pm 0.024$ & $1.1 \pm 2.4$ & $2.0 \pm 2.3$ & $2.7 \pm 2.0$ & $2.9^{+0.2}$ & 1.20 \\
\hline 23 & 1.795 & $1.188 \pm 0.004$ & $0.543 \pm 0.028$ & $0.0 \pm 2.7$ & $4.7 \pm 2.4$ & $5.7 \pm 3.0$ & $2.4^{-0.3}$ & 1.18 \\
\hline 24 & 1.769 & $1.349 \pm 0.004$ & $0.428 \pm 0.026$ & $5.6 \pm 2.2$ & $3.6 \pm 2.1$ & $0.3 \pm 3.0$ & $5.1_{-0.0}^{+0.3}$ & 1.06 \\
\hline 25 & 1.606 & $1.229 \pm 0.004$ & $0.366 \pm 0.024$ & $3.4 \pm 2.0$ & $1.3 \pm 2.0$ & $1.4 \pm 3.0$ & $4.4_{-0.5}^{+0.5}$ & 1.20 \\
\hline 26 & 1.247 & $0.925 \pm 0.004$ & $0.284 \pm 0.023$ & $1.22 \pm 1.9$ & $4.3 \pm 1.8$ & $3.6 \pm 2.0$ & $4.4_{-0.6}^{+0.5}$ & 0.96 \\
\hline 27 & 1.030 & $0.532 \pm 0.003$ & $0.399 \pm 0.030$ & $9.2 \pm 2.6$ & $2.2 \pm 2.7$ & $0.9 \pm 2.0$ & $4.3^{+0.5}$ & 0.82 \\
\hline 28 & 1.323 & $0.921 \pm 0.002$ & $0.365 \pm 0.020$ & $1.9 \pm 1.9$ & $0.0 \pm 1.9$ & $3.2 \pm 2.0$ & $4.3_{-0.4}^{+0.5}$ & 0.87 \\
\hline \multicolumn{9}{|c|}{$\begin{array}{r}0.505 \pm 0.020 \\
2006 \text { Suzaku }\end{array}$} \\
\hline 29 & 1.325 & $0.944 \pm 0.009$ & $0.297 \pm 0.020$ & $2.2 \pm 2.4$ & $4.7 \pm 2.4$ & $6.3 \pm 1.7$ & $2.9^{+0.1}$ & 1.17 \\
\hline 30 & 0.768 & $0.441 \pm 0.004$ & $0.215 \pm 0.016$ & $4.5 \pm 1.8$ & $5.0 \pm 1.8$ & $8.4 \pm 1.3$ & $2.9^{-0.1}+9$ & 0.99 \\
\hline $31^{2}$ & 1.238 & $0.707 \pm 0.009$ & $0.407 \pm 0.020$ & $4.1 \pm 1.8$ & $2.8 \pm 1.8$ & $1.0 \pm 1.3$ & $1.3^{-0.3}$ & 1.16 \\
\hline 32 & 2.344 & $1.888 \pm 0.020$ & $0.443 \pm 0.050$ & $0.0 \pm 1.8$ & $0.0 \pm 8.0$ & $1.5 \pm 8.8$ & $2.7_{-0.8}^{+1.3}$ & 0.71 \\
\hline
\end{tabular}

Notes: (1) XMM fits have 133 degrees of freedom, Suzaku fits have 145 degrees of freedom. (2) Slice 31 required $N_{\mathrm{H}}(2)$ free, with value $3.4_{-1.5}^{+1.1} \times$ $10^{22} \mathrm{~cm}^{-2}$. 
T. J. Turner et al.: Time-resolved spectroscopy of Mrk 766, Online Material p 4

Table A.2. Values of fitted parameters for the "absorption" model, in each time slice, as described in the text.

\begin{tabular}{|c|c|c|c|c|c|c|c|c|}
\hline \multicolumn{9}{|c|}{ Partial covering model } \\
\hline \multirow{2}{*}{$\begin{array}{l}\text { Time } \\
\text { Slice } \\
\end{array}$} & Total & Direct & Absorbed & $6.97 \mathrm{keV}$ & $7.30 \mathrm{keV}$ & $6.4 \mathrm{keV}$ & \multirow{2}{*}{$\begin{array}{c}N_{\mathrm{H}}(1) \\
/ 10^{22} \mathrm{~cm}^{-2} \\
\end{array}$} & \multirow[t]{2}{*}{$\chi_{r}^{21}$} \\
\hline & \multicolumn{3}{|c|}{ 2-10 keV flux $/ 10^{-11} \mathrm{erg} \mathrm{cm}^{-2} \mathrm{~s}^{-1}$} & \multicolumn{3}{|c|}{ Line photon flux $/ 10^{-6} \mathrm{~cm}^{-2} \mathrm{~s}^{-1}$} & & \\
\hline & & & & \multicolumn{3}{|c|}{$2000 X M M$} & & \\
\hline 1 & 1.422 & $1.079 \pm 0.010$ & $0.348 \pm 0.040$ & $2.2 \pm 2.0$ & $3.9 \pm 1.8$ & $0.0 \pm 5.0$ & $0.0_{-0.0}^{+0.5}$ & 1.04 \\
\hline \multirow[t]{2}{*}{2} & 1.617 & $1.446 \pm 0.024$ & $0.173 \pm 0.050$ & $0.9 \pm 2.9$ & $1.9 \pm 2.7$ & $0.0 \pm 5.0$ & $0.4_{-0.4}^{+0.0}$ & 1.13 \\
\hline & \multicolumn{6}{|c|}{$2001 X M M$} & & \\
\hline 3 & 2.797 & $2.793 \pm 0.006$ & $0.000 \pm 0.036$ & $0.0 \pm 2.8$ & $3.4 \pm 2.5$ & $4.5 \pm 5.2$ & $4.0_{-40}^{+100.0}$ & 0.87 \\
\hline 4 & 2.556 & $2.507 \pm 0.007$ & $0.035 \pm 0.030$ & $4.1 \pm 2.7$ & $2.1 \pm 2.5$ & $7.8 \pm 5.1$ & $0.69_{-0 .}^{+1.0}$ & 0.87 \\
\hline 5 & 2.715 & $2.682 \pm 0.007$ & $0.035 \pm 0.030$ & $0.0 \pm 2.7$ & $0.0 \pm 2.5$ & $4.6 \pm 5.0$ & $0.28_{-0.7}^{+8.8}$ & 0.79 \\
\hline 6 & 2.859 & $2.794 \pm 0.004$ & $0.061 \pm 0.030$ & $2.3 \pm 2.9$ & $3.8 \pm 2.6$ & $7.2 \pm 5.0$ & $0.36_{-0.4}^{+2.7}$ & 0.83 \\
\hline \multirow[t]{2}{*}{7} & 1.843 & $1.610 \pm 0.007$ & $0.238 \pm 0.026$ & $0.0 \pm 2.5$ & $3.4 \pm 2.5$ & $0.0 \pm 5.0$ & $0.36_{-0.4}^{+2.8}$ & 0.83 \\
\hline & \multicolumn{6}{|c|}{$2005 X M M$} & & \\
\hline 8 & 0.550 & $0.253 \pm 0.003$ & $0.307 \pm 0.017$ & $6.4 \pm 1.3$ & $4.4 \pm 1.1$ & $2.4 \pm 2.9$ & $9.34_{-1.3}^{+1.7}$ & 1.01 \\
\hline 9 & 0.930 & $0.366 \pm 0.003$ & $0.578 \pm 0.017$ & $11.0 \pm 1.6$ & $9.4 \pm 1.4$ & $3.5 \pm 3.5$ & $1.47_{-0.3}^{+0.7}$ & 1.29 \\
\hline 10 & 0.738 & $0.309 \pm 0.003$ & $0.435 \pm 0.018$ & $6.4 \pm 1.4$ & $0.0 \pm 1.5$ & $3.0 \pm 3.2$ & $5.39_{-0.0}^{+0.0}$ & 0.80 \\
\hline 11 & 0.572 & $0.217 \pm 0.004$ & $0.361 \pm 0.027$ & $4.0 \pm 2.4$ & $1.7 \pm 2.2$ & $0.9 \pm 4.4$ & $12.28_{-0.1}^{+0.1}$ & 1.07 \\
\hline 12 & 0.779 & $0.447 \pm 0.004$ & $0.339 \pm 0.018$ & $5.8 \pm 1.5$ & $5.1 \pm 1.4$ & $4.8 \pm 3.3$ & $3.85_{-0.5}^{+0.6}$ & 1.11 \\
\hline 13 & 1.405 & $1.129 \pm 0.005$ & $0.280 \pm 0.021$ & $5.7 \pm 1.9$ & $4.8 \pm 1.7$ & $6.2 \pm 4.0$ & $0.05_{-0.1}^{+1.1}$ & 1.26 \\
\hline 14 & 1.218 & $1.006 \pm 0.004$ & $0.213 \pm 0.020$ & $2.5 \pm 1.8$ & $4.5 \pm 1.6$ & $4.8 \pm 3.7$ & $0.65_{-0.7}^{+1.7}$ & 1.08 \\
\hline 15 & 1.159 & $0.955 \pm 0.004$ & $0.206 \pm 0.023$ & $3.7 \pm 1.9$ & $3.9 \pm 1.7$ & $5.2 \pm 4.0$ & $1.25_{-0.5}^{+3.8}$ & 0.93 \\
\hline 16 & 1.303 & $1.097 \pm 0.005$ & $0.202 \pm 0.021$ & $0.0 \pm 1.9$ & $1.3 \pm 1.7$ & $3.0 \pm 3.7$ & $0.16_{-0.2}^{+2.9}$ & 1.01 \\
\hline 17 & 1.552 & $1.434 \pm 0.005$ & $0.122 \pm 0.025$ & $1.1 \pm 2.0$ & $5.3 \pm 1.8$ & $3.6 \pm 3.7$ & $3.50_{-3.0}^{+2.6}$ & 0.97 \\
\hline 18 & 1.450 & $1.157 \pm 0.005$ & $0.289 \pm 0.022$ & $0.3 \pm 2.1$ & $0.0 \pm 2.0$ & $0.0 \pm 4.0$ & $0.24_{-0.2}^{+0.7}$ & 0.77 \\
\hline 19 & 1.266 & $1.063 \pm 0.004$ & $0.202 \pm 0.025$ & $0.0 \pm 2.2$ & $2.6 \pm 1.9$ & $1.2 \pm 5.0$ & $3.26_{-1.5}^{+1.5}$ & 0.93 \\
\hline 20 & 1.477 & $1.293 \pm 0.004$ & $0.187 \pm 0.023$ & $1.9 \pm 2.0$ & $2.4 \pm 1.8$ & $1.2 \pm 4.0$ & $1.36_{-1.4}^{+2.6}$ & 0.93 \\
\hline 21 & 1.731 & $1.641 \pm 0.004$ & $0.092 \pm 0.026$ & $0.1 \pm 2.1$ & $3.0 \pm 1.9$ & $1.4 \pm 3.8$ & $4.85_{-48}^{+9.11}$ & 0.79 \\
\hline 22 & 1.960 & $1.579 \pm 0.004$ & $0.386 \pm 0.024$ & $2.1 \pm 2.4$ & $5.1 \pm 2.1$ & $1.2 \pm 4.0$ & $0.05_{-0.0}^{+0.6}$ & 1.06 \\
\hline 23 & 1.797 & $1.391 \pm 0.004$ & $0.410 \pm 0.028$ & $0.5 \pm 2.7$ & $7.8 \pm 2.3$ & $2.9 \pm 4.0$ & $0.05_{-0.0}^{+0.5}$ & 1.12 \\
\hline 24 & 1.774 & $1.590 \pm 0.004$ & $0.193 \pm 0.026$ & $4.0 \pm 2.1$ & $4.5 \pm 2.0$ & $0.0 \pm 5.0$ & $3.16_{-20}^{+1.3}$ & 0.78 \\
\hline 25 & 1.609 & $1.434 \pm 0.004$ & $0.180 \pm 0.024$ & $2.4 \pm 2.0$ & $2.9 \pm 1.9$ & $0.0 \pm 5.0$ & $0.05_{-0.0}^{+2.0}$ & 0.94 \\
\hline 26 & 1.249 & $1.089 \pm 0.004$ & $0.162 \pm 0.023$ & $0.5 \pm 1.9$ & $5.3 \pm 1.7$ & $3.1 \pm 5.0$ & $1.84_{-1.8}^{+2.2}$ & 0.69 \\
\hline 27 & 1.031 & $0.626 \pm 0.003$ & $0.408 \pm 0.030$ & $8.9 \pm 2.6$ & $3.7 \pm 2.5$ & $8.3 \pm 5.0$ & $2.82_{-0.3}^{+0.6}$ & 0.82 \\
\hline \multirow[t]{2}{*}{28} & 1.325 & $1.073 \pm 0.002$ & $0.252 \pm 0.020$ & $1.5 \pm 1.9$ & $1.3 \pm 1.8$ & $1.6 \pm 3.6$ & $0.83_{-0.8}^{+3.0}$ & 0.74 \\
\hline & \multicolumn{6}{|c|}{2006 Suzaku } & & \\
\hline 29 & 1.316 & $1.160 \pm 0.009$ & $0.167 \pm 0.020$ & $4.5 \pm 2.5$ & $7.9 \pm 2.2$ & $2.5 \pm 5.2$ & $0.0_{-0.6}^{+0.9}$ & 1.38 \\
\hline 30 & 0.764 & $0.564 \pm 0.004$ & $0.215 \pm 0.016$ & $7.9 \pm 1.9$ & $7.9 \pm 1.7$ & $2.8 \pm 4.1$ & $0.0_{-0.0}^{+0.0}$ & 1.40 \\
\hline $31^{2}$ & 1.238 & $0.801 \pm 0.009$ & $0.444 \pm 0.020$ & $5.8 \pm 2.3$ & $4.7 \pm 2.1$ & $0.0 \pm 4.9$ & $5.0_{-07}^{+0.6}$ & 1.14 \\
\hline 32 & 2.339 & $2.264 \pm 0.020$ & $0.077 \pm 0.050$ & $0.4 \pm 8.1$ & $1.4 \pm 8.0$ & $0.0 \pm 14.0$ & $0.0_{-0.0}^{+4.9}$ & 0.69 \\
\hline
\end{tabular}

Notes: (1) XMM fits have 133 degrees of freedom, Suzaku fits have 145 degrees of freedom. (2) Slice 31 required $N_{\mathrm{H}}(2)$ free, with value $0.0<$ $0.5 \times 10^{22} \mathrm{~cm}^{-2}$. 